\title{
El aprendizaje colaborativo como estrategia didáctica para el mejoramiento de la Comprensión lectora ${ }^{1}$ \\ Collaborative learning as didactic strategies for the improvement of reading comprehension
}

DOI: http://dx.doi.org/10.17981/cultedusoc.9.3.2018.13

Artículo de investigación. Fecha de recepción: 15/06/2018. Fecha de aceptación: 27/11/2018

Paulina Esquivel';

Fernando Villa; Gabriel Guerra; Carmenza Guerra y Eusebio Rangel ${ }^{3}$

IED Nuestra señora del Carmen. Sede -principal (Colombia)

titamelanie@hotmail.com

Para citar este artículo:

Esquivel, P., Villa. F., Guerra, G., Guerra, C. y Rangel, E. (2018). El aprendizaje colaborativo como estrategia didáctica para el mejoramiento de la Comprensión lectora. Cultura. Educación y Sociedad 9(3), 105-112. DOI: http://dx.doi.org/10.17981/cultedusoc.9.3.2018.13

\section{Resumen}

El aprendizaje colaborativo se convierte en un instrumento de saber práctico que favorece los espacios de apropiación de conocimiento no solo para los estudiantes sino también para la comunidad educativa en general. La presente investigación busca mostrar los procesos de comprensión lectora, en los estudiantes de la Institución Educativa Nuestra Señora del Carmen, fomentando herramientas didácticas como el aprendizaje colaborativo que potencie la motivación por la lectura. El presente estudio se realiza desde una mirada cuantitativa, desde un alcance descriptivo. La población intervenida fueron estudiantes de básica primaria. Como resultados se permitió observar que el trabajo en grupo u colaborativo no solo se puede incrementar el rendimiento académico, sino que además presenta otras virtudes como que el estudiante aprende a trabajar en equipo y el modelo de aprendizaje interactivo supone que las experiencias de aula deben superar el aprendizaje memorístico y mecanicista.

Palabras clave: Estrategia Didáctica, Aprendizaje Colaborativo, Comprensión Lectora.

\section{Abstract}

Collaborative learning becomes an instrument of practical knowledge that favors knowledge appropriation spaces not only for students but also for the educational community in general. This research seeks to show the processes of reading comprehension, in the students of the Educational Institution of Our Lady of Carmen, fostering teaching tools such as collaborative learning that enhances the motivation for reading. The present study is carried out from a quantitative perspective, from a descriptive scope. The population intervened were elementary school students. As results, it was possible to observe that group or collaborative work can not only increase academic performance, but also presents other virtues such as that the student learns to work in a team and the interactive learning model assumes that classroom experiences must overcome the memoristic and mechanistic learning.

Keywords: Didactic strategy, Collaborative Learning, Reading Comprehension.

1 Este artículo ha sido derivado del Programa de Fortalecimiento de la Cultura Ciudadana y Democrática CT+I a través de la IEP apoyada en TIC en el Departamento de Magdalena: CICLON.

2 Líder del grupo de investigación "generadores del saber".

3 Docentes de la IED Nuestra señora del Carmen, sede principal. Grupo de Investigación "Generadores del Saber".

- The author; licensee Universidad de la Costa - CUC.

Cultura, Educación y Sociedad vol. 9 no. 3, pp. 105-112. Diciembre, 2018

Barranquilla. ISSN 2389-7724 Online 


\section{Introducción}

La razón que impulsa la presente investigación sostiene un enorme valor desde la perspectiva de cambiar elementos presentes en la vida escolar de la población objetivo en este caso los estudiantes de la Institución Educativa Nuestra Señora del Carmen por medio de las IEP y las TICS, que servirán como premisas fundamentales en las costumbres de mejoramiento de la capacidad lectora de los estudiantes, la primera tiene que ver con los resultados de la institución en los niveles de comprensión lectora.

Desde el espacio que se busca abrir y comprender dentro de esta investigación y que enmarca el proceso educativo, es esencialmente que el estudiante desde su razonamiento, pueda adquirir las capacidades que lo lleven a comprender el contexto y el mundo dónde vive, desde esta premisa surgen niveles de conocimientos, que ayudan a promover competencias indispensables para desenvolverse en el intrincado sentido de la sociedad de hoy, por eso lo manifestado por Carrasco, (2001) donde afirma "Leer es un proceso en el cual se usan estrategias para relacionar la nueva información con los esquemas socialmente adquiridos, el desarrollo de la capacidad de autorregulación e incidir en las creencias que tiene el alumno" (p.138).

El centro de todo proceso lector es la capacidad que tenga los estudiantes para llevar a cabo su compromiso con el texto y afrontarlo, desde una orientación planeada pero libre por parte del docente y es uno de los puntos más significativos para saber que las competencias lectoras deben ser promovidas desde el aula no olvidando el enorme recurso que tiene el contexto cultural en el desarrollo de competencias lectoras.

Desde las razones de las competencias, las pruebas y la realidad vivida hoy por los estudiantes en caso específico del Departamento del Magdalena, donde no se puede desconocer que por sus antecedentes anuales muestran que se está en términos de competencias lectoras, así lo determina el Índice Sintético de la Calidad 2015, dónde se avanzó un poco en la primaria pero aún en la básica secundaria se tiene falencias.

Desde otra mirada y buscando el mejoramiento de la capacidad escritural y lectora del estudiante, es indispensable saber según Baena (1992) que la significación, el proceso por medio del cual se le da sentido a una experiencia, ocurriendo una metamorfosis en relación a las categorías conceptuales, prácticas y culturales.

Por las anteriores razones, la lengua se convierte en una progresión verbal que se adquiere con la integración del individuo a la sociedad y la perfecciona desde otros ámbitos y hábitos entre ellos el educativo. Es por eso que los fenómenos de la lectura y la escritura son un legado cultural que debe ser enseñado y contextualizado porque cada proceso de esto a pesar de su generalización tiene causas particulares en la lectura y la escritura. La cultura como dimensión de análisis para los procesos lectores puede estar asociada a la vulnerabilidad como variable clave en el comportamiento de las comunidades. En este sentido, Romero y Contreras (2015) destacan que la vulnerabilidad y las situaciones de conflicto determinan formas comportamentales, de tal forma que se podría inferir su importancia al momento de consolidar un perfil que privilegie el desarrollo de competencias lectoras.

En este contexto referencial, la propuesta tiene como población objetivo a los estudiantes del Grado Sexto de la Educación Básica secundaria de la institución educativa Nuestra Señora del Carmen del municipio de Guamal, Departamento del Magdalena, de igual manera a padres de familia y docentes responsables. 


\section{Métodos de enseñanza}

Actualmente los métodos de enseñanza han sufrido significativas transformaciones, dentro de los cuales cabe señalar la reorientación de los patrones educativos, la adquisición de nuevas tecnológicas y la articulación de las competencias en los modelos de aprendizaje; esto con el fin de afianzar los procesos de enseñanza aprendizaje y responder a las demandas de la sociedad actual. (Marín; Inciarte; Hernández y Pitre. 2017).

Según, Abreu (1994), concibe el método de enseñanza como " un elemento movilizador de los componentes cognitivos, psicomotrices y afectivos de la personalidad del estudiante en formación, en una situación concreta de aprendizaje para estimular el desarrollo individual y grupal"; esta concepción, genera la idea que es necesario que el docente integre en su planeación metodológica, acciones que movilicen en el componente cognitivo el desarrollo de habilidades y competencias, que sea un diseñador de estrategias que orienten en sus estudiantes un pensamiento basado en el orden de las ideas, critico, reflexivo, que argumente sus posiciones y sea coherente en la toma de decisiones desde un análisis constructivo.

Siguiendo la anterior apreciación, es importante tener en cuenta que, para alcanzar esta meta, el papel de la Educación Primaria es fundamental. Es durante la Primaria en donde los estudiantes aprenden a leer y a escribir. En esta etapa los niños comienzan a utilizar el lenguaje escrito como una herramienta de comunicación y de acceso al conocimiento. Es decir, que durante el ciclo de Básica Primaria, se consolidan aprendizajes de la lengua escrita y son capaces de utilizar este conocimiento en situaciones concretas, tanto comunicativas como investigativa. $\mathrm{Y}$, es así como "La competencia lectora bien desarrollada constituye uno de los requisitos para continuar aprendiendo, pero también es el resultado de haber aprendido." (Gómez, 2012: 24).

SEP, (2011) plantea como rasgos deseables en lo que respecta a las competencias de lectura para los estudiantes que terminan la secundaria debe ser capaz de comunicarse con claridad y fluidez utilizando un lenguaje oral y escrito así, como interactuar en distintos contextos sociales. Tener la capacidad de buscar, seleccionar, analizar, evaluar y utilizar la información proveniente de diversas fuentes. A lo que llamaríamos alfabetización informacional.

Echevarría, (2006); Feo, (2010) comentan que hoy por hoy, se reconoce a la alfabetización como un proceso soportado en el texto interactivo con una finalidad definida, y que depende del texto mismo y de la persona que lo lee. Teniendo en cuenta que el presente proyecto de investigación se fundamenta en las categorías de trabajo colaborativo, estrategia didáctica y comprensión lectora, se hace necesario abordar una serie de aspectos relevantes para el desarrollo del fundamento teórico.

Cuando se habla de competencia lectora, necesariamente se hace referencia a la comprensión a partir de una reflexión de textos escritos; con el fin de lograr desarrollar conocimiento y promover la participación activa del individuo en la sociedad. (OCDE, 2013).

\section{Trabajo Colaborativo}

Esta forma de trabajo se constituye en un modelo de aprendizaje dinámico donde la interacción entre sujetos juega un rol preponderante, motiva a la construcción social del conocimiento, por lo que requiere de aunar esfuerzos entre los participantes e integrar las diversas competencias presentes en cada uno de ellos de tal manera que pueda lograrse la meta propuesta. 
Como lo expresa Martín (2001) citado por Maldonado (2007), el trabajo colaborativo es una forma de interacción entre personas que donde el respeto por el aporte individual que hace cada miembro es importante.. En este sentido, este modelo de aprendizaje interactivo supone que las experiencias de aula deben superar el aprendizaje memorístico y mecanicista.

Así como lo afirman Ramírez y Rojas (2014), "Fomentar el trabajo colaborativo implica un cambio en la cultura escolar, en las prácticas de enseñanza/aprendizaje y en los procesos de evaluación; actualmente, la pedagogía y en sí, la educación, buscan que entre los estudiantes se fomente la colaboración entendida como una forma legítima y eficiente de producción tanto de conocimiento como de contenidos para que así, en la escuela, los estudiantes aprendan unos de otros".

Esto implica que los ambientes de aprendizaje enriquecidos por la aplicación del trabajo colaborativo permiten que los estudiantes sean más activos en la medida que buscan la consecución de objetivos comunes desarrollando las habilidades y potencialidades de trabajo, asumen una democracia participativa asumiendo roles de grupo. En este sentido, autores como Romero y Contreras (2015), destacan la necesidad de que los sujetos en el ámbito de las interacciones sociales puedan fortalecer sus proyectos de vida a partir de las capacidades que permitan su participación asertiva en la toma de decisiones.

El trabajo colaborativo como estrategia didáctica no implica "hacerle el trabajo al otro", ni descansar en que el resto se hará cargo, (Bugueño y Barros, 2008:1), El trabajo colaborativo, va más allá de la responsabilidad de cada miembro del equipo, en esencia lo que se valora es el compromiso de cada uno de ellos en las etapas de planificación, análisis y evaluación de lo que se hace, donde cada miembro cumple responsablemente con el rol que le corresponde.

\section{Estrategias Didácticas}

Considerar el trabajo colaborativo como estrategia didáctica conlleva a reflexionar sobre la conceptualización de la estrategia didáctica en el aula.

Por consiguiente, Carrasco (1997) haciendo alusión a estos dos conceptos, se refiere que las estrategias en el campo didáctico son las diversas formas de abordar el conocimiento empleadas por el profesor para garantizar el aprendizaje de los discentes.

Sin embargo, se resalta que toda esta diversidad de formas implementadas difícilmente es expuestas o mostradas con exactitud debido a que la didáctica es un arte y como tal su interpretación es muy particular porque se da dependiendo de cada docente.

Así mismo, los autores Velasco y Mosquera (2010); Magallanes (2011), definen la estrategia didáctica como la organización desde una planeación, de los procesos de enseñanza y del aprendizaje, para ello se hace necesario la escogencia por parte del docente de las herramientas, instrumentos y activdades necesarias para el logro de los propositos de formación planteados, lleva consigo la toma de decisiones a partir de la reflexión para la consecudión de los mismos.

Del mismo modo, Carrasco (2004) senala que existen tres tipos de estrategias didácticas: Los métodos didácticos, técnicas de enseñanza y procedimientos didácticos. En donde los métodos los define como la organización racional y práctica de los medios, técnicas y procedimientos de enseñanza, para dirigir el aprendizaje del estudiante, es decir, ordenar y concretar un conjunto de reglas que permitan obtener los resultados esperados. 


\section{Comprensión Lectora}

Gutiérrez y Montes de Oca (2007), consideran la comprensión lectora como un proceso dinamico de interacción comunicativa entre los que participan del proceso (texto-lector), el lector comprende cuando logra interiorizar y le da significado al contenido leído.

Carrillo Torea (2007) considera que la lectura como experiencia es individual, y su prática constante es la que hace que el lector se acerque más al gusto por ella. La comprensión de un texto exige el desarrollo de procesos cognitivos como la interpretación y representación mental de cada uno de los significados a partir de la información identificada en el texto articulada con los presaberres que posee el lector, sin perder de vista el propósito de la lectura y el contexto en el que se lleva a cabo.

La representación mental de la que se habla es "construida de manera procesual haciendo inferencias y reelaboraciones de la representación mental de lo comprendido en el texto" (Parodi, 2009, p. 39).

En consecuencia, la comprensión textual puede considerarse como un proceso cognitivo complejo, que se "materializa" o concretiza en un discurso escrito resultados de los procesos cognitivos mencionados anteriormente y que el lector de manera consciente activa y desarrollo estos procesos relacionándolo con lo que conoce para otorgarle un significado propio.

Aunado a esto, se evidencia que en la comprensión también intervienen variables asociadas al lector como son: los conocimientos previos, visión del mundo; relacionadas con el contexto: el a mbiente, nivel de escolaridad, situaciones del contexto y las relacionadas con el texto: tipo, estructura textual y rasgos lingüísticos, entre otros.

\section{Metodología}

Este estudio se llevará a cabo desde una mirada Cuantitativa, de alcance descriptivo, orientado a discriminar las propiedades, las características de personas, colectivos, procesos, objetos o cualquier otro fenómeno que se someta a un análisis.

\section{Población y muestra}

La población estudio son los estudiantes del grado $6^{\circ} \mathrm{B}$ de la institución educativa Nuestra Señora del Carmen. Con una muestra de 28 estudiante de $6 \mathrm{~B}$.

\section{Técnicas e instrumentos de recolección de la información}

Dado el tipo de investigación cuantitativa, se decide utilizar como técnicas de recolección: la encuesta. De manera que la información es registrada en un instrumento tipo cuestionario- escala tipo Likert que permiten su clasificación y nos proporciona facilidad para su análisis e interpretación.

El diseño de la Encuesta se formuló de tal manera que se obtuviera información precisa sobre el objeto de investigación, descubriendo la información respecto a los sujetos de estudio en relación con el trabajo colaborativo para fortalecer la comprensión lectora.

\section{Procedimiento}

Fase (1): Identificación de la problemática a estudiar.

Fase (2): Definición de la población y muestra.

Fase (3): Definición de las variables de estudio.

Fase (4): Fundamentación teórica.

Fase (5): Escogencia de las técnicas e instrumentos de recolección de información. 
Fase (6): Aplicación de los instrumentos. Fase (7): Análisis y procesamiento de los datos.

Fase (8): Generación de resultados.

Fase (9): Socialización y discusión de los hallazgos encontrados.

\section{Resultados}

A continuación se presentan los resultados tomando en cuenta las preguntas de la escala.

Con relación a la primera pregunta que hace alusión a la forma de trabajar 20 estudiantes contestan que les gusta el trabajo grupal, 5 estudiantes el trabajo en casa y 3 estudiantes el trabajo individual.

Tomando en cuenta la segunda pregunta sobre el tema de interés que predomina entre los estudiantes del grado $6 \mathrm{~b}$ es relacionado con el miedo, seguido de los temas relacionados con el medio ambiente, también les interesan temas de leyendas, de valores y cuentos y de tecnología.

La pregunta 3 que hace mención a la cantidad de libros leídos en el último año escolar tiempos 5 estudiantes han leído 1libro; 5 estudiantes han leído 2 libros, 7 estudiantes han leído 3 libros y 11 estudiantes han leído más de 4libros.

En cuanto a la pregunta 4 sobre cuántos libros han comprado en tu casa este año, los estudiantes contestan: 6 han comprado $1 ; 7$ han comprado $2 ; 5$ han comprado $3 ; 10$ han comprado más de 4 .

La pregunta 5 sobre si los profesores asignan actividades en grupo 2 estudiantes refieren que siempre; 26 refieren que algunas veces y 1 no respondió

Finalmente la pregunta que hace referencia al gusto sobre la lectura con recursos físicos o digitales 14 estudiantes contestó que les gusta leer por medio físico, 11 por medios virtuales y 3 por ambos medios.

\section{Conclusiones}

De acuerdo a los resultados suministrados con anterioridad se puede inferir que modelo de aprendizaje interactivo supone que las experiencias de aula deben superar el aprendizaje memorístico y mecanicista.

El docente debe conocer y apropiarse de la estrategia de trabajo colaborativo al momento de aplicarla en el aula de clases, atendiendo al desarrollo cognitivo y desarrollo social propio de la edad escolar de los estudiantes. Además, identificar las características de esta estrategia, aspectos que la integran y ventajas, debido a que el trabajo colaborativo es considerado como una estrategia de interacción social en la que los participantes logran un aprendizaje significativo, desarrollan habilidades de tipo cognitivo, además que tributa a fortalecer competencias actitudinales encauzadas al desarrollo integral de la persona (Magallanes, 2011).

Finalmente, el trabajo en grupo u colaborativo no solo se puede incrementar el rendimiento académico, sino que además presenta otras virtudes como que el estudiante aprende a trabajar en equipo, dar y recibir críticas, a planificar, a guiar y evaluar su actividad individual.

\section{Referencias}

Abreu, R. (1994). Modelo Teórico básico de la pedagogía profesional. La Habana: CEPROF. ISPETP.

Avendaño, Cortés O, Guerrero H. ( 2015) Competencias sociales y tecnologías de la información y la comunicación como factores asociados al desempeño en estudiantes de básica primaria con experiencia de desplazamiento forzado Diversitas: Perspectivas en Psicología, 11(1). 13-36. 
Brufee, K. (1994). Collaborative Learning: Higher Education, Interdependence and the Authority of Knowledge. Baltimore: Johns Hopkins Press.

Brufee, K. (1995). Sharing our toys: Cooperative learning versus collaborative learning. Journal Change: The Magazine of Higher Learning, 27(1). 12-18. https://doi.org/10.1080/00091383.199 5.9937722

Bugueño, X. y Barros, C. (2008). Fichas docentes. Formación e equipos de trabajo colaborativo. Valoras UC. Bogotá: Ministerio de Educación.

Cabero, J. (2003). Principios pedagógicos, psicológicos y sociológicos del trabajo colaborativo: su proyección en la telenseñanza. En, Redes de comunicación en la enseñanza: las nuevas perspectivas del trabajo corporativo (129-156). Paidós Ibérica.

Cabero., J. (2003). Principios pedagógicos, psicológicos y sociológicos del trabajo colaborativo: su proyección en la tele enseñanza. En, Martínez, F. (Compiladores), Redes de comunicación en la enseñanza. Las nuevas perspectivas de trabajo corporativo. Barcelona: Paidós.

Carrasco, J. (2004). Una didáctica para hoy: cómo enseñar mejor. Madrid: Ediciones Rialp.

Carrasco, J. (1997). Técnicas y recursos para el desarrollo de las clases. Madrid: Rialp.

Castellaro, M., y Dominino, M. (2011). El proceso colaborativo en niños de escolaridad inicial y primaria. Una revisión de trabajos empíricos. Revista Intercontinental de Psicología y Educación. 13(2). 119-145.

Cerda, H. (1998). Los elementos de la investigación. Bogota: El Buho.
Chavarria, M. (1985). Desarrollo ya atención en niños de 7 a 12 años. Sanjose costa rica: Editorial universidad estatal a distancia.

Collazos, C., Guerrero, L., \& Vergara, A. (2001). Aprendizaje Colaborativo: un cambio en el rol del profesor. In Proceedings of the 3rd Workshop on Education on Computing, Punta Arenas, Chile.

Curiche, D. (2015). Desarrollo de habilidades de pensamiento crítico por medio de aprendizaje basado en problemas y aprendizaje colaborativo mediado por computador. Tesis inédita de maestría. Universidad de Chile, Santiago de Chile.

Díaz, F, \& Hernández, G. (1998). Estrategias docentes para un aprendizaje significativo. Editorial Mc Graw Hill. México.

Feo, R. (2010). Orientaciones Básicas Para El Diseño De Estrategias Didacticas. Tendencias Pedagogicas(16), 219-236.

Godat, M. (2012). Collaborative Learning and Critical Thinking in Technologyenhanced Environments. Tesis para optar el título de Doctor en Filosofia en Universidad de Tecnología de Queensland.

Gros, B (2013). Aprender y enseñar en colaboración. En Aprender en Red. Suárez, C. y Gros B. Editorial UOC.

Marín, F., Inciarte, A., Hernández, H., y Pitre, R. (2017). Estrategias de las instituciones de educación superior para la integración de las tecnologías de la información y la comunicación y de la innovación en los procesos de enseñanza. Un estudio en el distrito de Barranquilla, Colombia. Form. Univ. [online]. 10(6). 29-38. http://dx.doi.org/10.4067/ S0718-50062017000600004. 
Romero-Acosta, K. y Contreras, E. (2015). Revisión teórica sobre el post-conflicto: una oportunidad para empoderar a mujeres víctimas de desplazamiento. $\mathrm{Cul}$ tura Educación y Sociedad, 6(1). Recuperado de https://revistascientificas.cuc.edu.co/culturaeducacionysocie$\mathrm{dad} /$ article/view/757 